\title{
Microwave irradiation and quasiparticles in a superconducting double dot
}

\author{
N. J. Lambert, ${ }^{1, *}$ A. A. Esmail, ${ }^{1}$ F. A. Pollock, ${ }^{2}$ M. Edwards, ${ }^{1}$ B. W. Lovett, ${ }^{3}$ and A. J. Ferguson ${ }^{1}$ \\ ${ }^{1}$ Microelectronics Group, Cavendish Laboratory, University of Cambridge, Cambridge CB3 OHE, United Kingdom \\ ${ }^{2}$ School of Physics \& Astronomy, Monash University, Clayton, Victoria 3800, Australia \\ ${ }^{3}$ SUPA, School of Physics and Astronomy, University of St Andrews, St. Andrews KY16 9SS, United Kingdom
}

(Received 21 December 2016; published 9 June 2017)

\begin{abstract}
We study the interaction of the charge states of a superconducting double dot, comprising two superconducting islands coupled by a Josephson junction, with microwaves between 2 and $55 \mathrm{GHz}$. We observe resonant transitions between even-parity charge states at relatively low frequencies and breaking of Cooper pairs under higherfrequency irradiation, allowing our device to act as a click detector for microwave photons. By applying a magnetic field and tuning the pair-breaking energy, we perform spectroscopy on the environment in our cryostat and determine the temperature of a nonequilibrium photon bath. Finally, we exploit the band structure of our device to break Cooper pairs dependent on the symmetry of the initial Cooper pair state.
\end{abstract}

DOI: 10.1103/PhysRevB.95.235413

\section{INTRODUCTION}

The interaction between microwave light and superconducting devices underpins studies of fundamental quantum mechanics [1] and technologies for a range of applications [2]. The interaction can be coherent, leading to Rabi oscillations between the field and a superconducting qubit [3]. A family of superconducting qubits exploiting different degrees of freedom [4-7] has been developed in order to explore and exploit this for quantum computing [8,9], microwave detection [10], or, more generally, for studies of the strong coupling between light and matter [11]. Alternatively, the interaction may involve the incoherent absorption of a photon and creation of Bogoliubov quasiparticle excitations in the superconductor, a process which can form the basis of microwave detection technology [12-14]. A device capable of single-microwave-photon detection is desirable for a range of fields including metrology [15] and millimeter-wave astrophysics [16]. It would also enable new approaches to quantum information processing that directly exploit quantum optics in the microwave regime. For example, a single-photon detector would allow entanglement generation by measurement $[17,18]$ at microwave frequencies, and the execution of linear optical quantum information protocols [19]. Such a detector would allow unprecedented measurement of the cryogenic environment on which quantum electronic devices rely [20,21].

A variety of technologies have been demonstrated for the detection of microwave frequencies, including transition-edge sensors [12], kinetic inductance detectors [13], bolometric detectors [22], and superconducting tunnel junctions [23]. However, unlike for visible radiation [24], single-photon detection in the microwave regime is extremely difficult to achieve because the energy of each photon is low. Tunnel junction detectors initially relied on the burst of current driven by a single photon breaking many Cooper pairs, only very recently evolving towards detection at the single-photon level [25]. Alternatively, a flux qubit coupled to a resonator [10] can be used for single-photon detection, although this approach requires complicated and precise control over the qubit.

\footnotetext{
*Corresponding author: n1249@ cam.ac.uk
}

In this paper, we discuss a simpler and more natural approach to photon detection by exploiting the relative ease with which Cooper pairs can be broken in a superconducting ground state, creating quasiparticle excitations. The Cooper pairs have a binding energy of $2 \Delta$, where $\Delta$ is the superconducting gap, and in bulk aluminium, $2 \Delta \approx 400 \mu \mathrm{eV} \approx 106 \mathrm{GHz}$. The fragility caused by this weak binding can also be troublesome; nonequilibrium quasiparticles in superconducting qubits can destroy the coherence of the system [26,27]. An understanding of this quasiparticle poisoning is important for further optimization of superconducting qubits [27-30].

We describe both the coherent and incoherent interaction of a superconducting double dot (SDD) with microwave photons. An SDD [Fig. 1(a)] comprises two superconducting islands tunnel coupled by a Josephson junction [31,32]. They are individually tunnel coupled to metallic leads via normal-insulator-superconductor junctions. Such a device is analogous to semiconductor double dots, which have been demonstrated as charge qubits [33], spin qubits [34-36], and single-photon detectors [37]. Control over these devices relies first on an understanding of the energetics of the spin and charge states [38,39], usually described as a function of the electrostatic gate voltages used to control the chemical potentials of the two dots. We therefore begin by describing the band structure of the SDD.

\section{THE BAND STRUCTURE OF A LOW CHARGING ENERGY SDD}

The energy-band structure and stability diagram of the charge states of an SDD depends on the competition between the various energy scales of the device and its environment. Increasing the charging energies of the islands $\left(E_{c}\right)$ and the temperature $\left(k_{B} T\right)$ makes quasiparticle states more preferable. Elsewhere [32], we discussed the effect of varying the ratio $\Delta / E_{c}$, but here we concentrate on a device in which $2 \Delta>$ $\left(E_{c}+E_{J}\right) / 2$ at $T=35 \mathrm{mK}$ and $B=0$, where $E_{J}$ is the Josephson energy of the middle tunnel junction and $B$ is the (constant) magnetic flux density in the plane of the device shown in Fig. 1(a). (A summary of the symbols used in this paper is given in Table I.) 
(a)
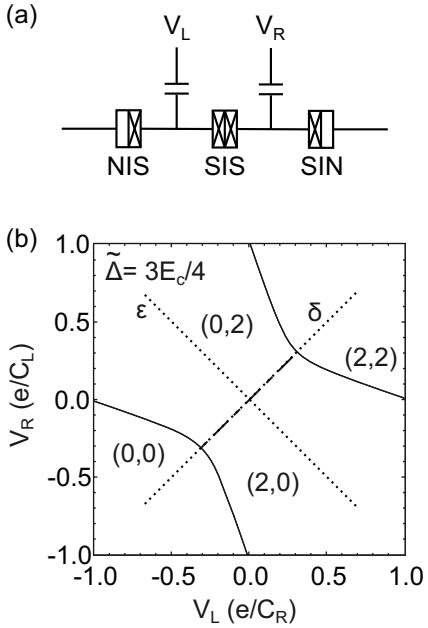

(c)

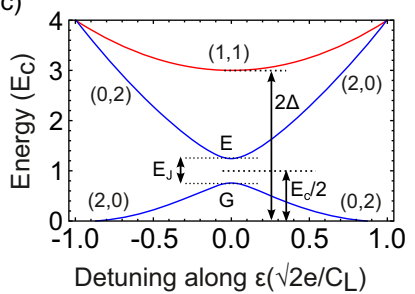

(d)

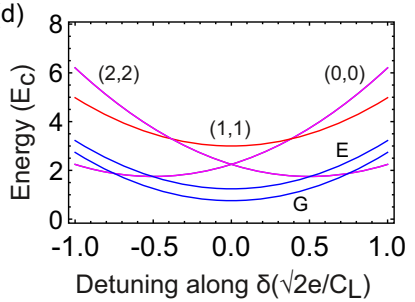

FIG. 1. An SDD and its energy-band structure, with $\tilde{\Delta} / E_{C}=$ $3 / 4$. (a) Schematic of double dot, showing a NISISIN structure. (b) Unit cell of the stability diagram of the SDD. Detuning axes $\epsilon$ and $\delta$ are shown. (c) Band structure along $\epsilon$ detuning, showing the anticrossing between the $(2,0)$ and $(0,2)$ states. (d) Band structure along $\delta$ detuning.

In this regime, the energy cost due to the superconducting gap for odd-parity charge states in which quasiparticles are present is large enough that they are never the ground state. The hexagonal stability diagram is therefore $2 e$ periodic, in analogy to Coulomb blockade in a superconducting single-electron transistor (SSET) [40]. In Fig. 1(b), we plot the modeled stability diagram with cross sections along the detuning axes $\delta$, along which the energy difference between the dots remains constant, and $\epsilon$, which corresponds to the movement of charge from one dot to the other. Charge states are numbered as $(m, n)$, where $m(n)$ is the charge on the left (right) dot relative to an

TABLE I. Selected symbols.

\begin{tabular}{ll}
\hline \hline Symbol & \multicolumn{1}{c}{ Meaning } \\
\hline & SDD dynamics \\
$\tilde{\Delta}$ & Reduced superconducting gap \\
$E_{c}$ & Device charging energy \\
$E_{J}$ & Josephson energy of middle tunnel junction \\
$p_{G(E)}$ & Population of symmetric (antisymmetric) \\
& Cooper pair state \\
$p_{q p}$ & Population of (1,1) state \\
$\Gamma_{G \rightarrow(1,1)(\rightarrow G)}$ & Rate for system to leave (enter) state $|G\rangle$ \\
$C_{Q}$ & Quantum capacitance \\
& Random telegraph signals \\
$y(t)$ & Signal level \\
$a_{0(1)}$ & Lower (upper) level of RTS \\
$a_{n}$ & Noise amplitude \\
$\Gamma_{0(1)}$ & Real transition rate from level $a_{0(1)}$ \\
$\Gamma_{0(1)}^{*}$ & Measured transition rate from level $a_{0(1)}$ \\
$\tau$ & Measurement bandwidth \\
$\omega$ & Angular frequency \\
\hline \hline
\end{tabular}

arbitrarily chosen even-parity state in units of $e$. The $(2,0)$ and $(0,2)$ states are coupled by the Josephson energy, and an anticrossing of size $E_{J}$ opens up between them [see Figs. 1(c) and $1(\mathrm{~d})]$. The eigenstates at $\epsilon=\delta=0$ are $\frac{1}{\sqrt{2}}(|2,0\rangle \pm|0,2\rangle$. We label these states $|G\rangle$ for the lower-energy symmetric state and $|E\rangle$ for the antisymmetric state.

Charge states with a quasiparticle present on one or both islands are pushed to higher energies due to the superconducting gap. There is an additional energy cost of $\tilde{\Delta}$ per quasiparticle $[32,40]$, with the $(1,1)$ state lying $2 \tilde{\Delta}-\left(E_{c}-E_{J}\right) / 2$ higher than the ground state at $\epsilon=\delta=0$. Here,

$$
\tilde{\Delta}=\Delta-k_{B} T \ln \left(N_{\mathrm{eff}}\right),
$$

where $N_{\text {eff }}$ is the number of microstates available to the quasiparticle. Typically, $N_{\text {eff }} \sim 10^{3}$ and accounts for the thermodynamic contribution to the free energy [40]. Singlequasiparticle states $[(1,0),(0,1)$, etc.] have an energy cost of $\tilde{\Delta}$ in addition to an electrostatic component at $\epsilon=\delta=0$ and are comparable in energy to $(1,1)$. We have previously determined that pair breaking happens predominantly to the $(1,1)$ state [31], while a cascade through single-quasiparticle states is involved in the relaxation of the system to the ground state. In this paper, we therefore concentrate on the $|G\rangle,|E\rangle$, and $(1,1)$ states.

The anticrossing between the even-parity states at $\epsilon=$ $\delta=0$ results in a quantum capacitance [31] of $C_{Q}=-\frac{d^{2} U}{d \epsilon^{2}}$, where $U$ is the energy of the eigenstate. This is similar to the additional capacitance observed due to the band structure curvature in SSETs and Cooper pair boxes [41,42], and the quantum capacitance observed at interdot charge transitions in semiconductor double quantum dots $[43,44]$. Close to $\epsilon=\delta=0$, we can therefore distinguish between three states of the SDD: the symmetric Cooper pair superposition with a capacitance contribution $+C_{Q}$, the antisymmetric Cooper pair superposition with a capacitance contribution $-C_{Q}$, and a state with at least one quasiparticle [including $(1,0),(0,1)$, etc.], which has no change in curvature and therefore no additional capacitance.

We will describe the transitions between charge states driven by microwave frequency radiation incident on the device. States $|G\rangle$ and $|E\rangle$ are eigenstates of the system and so the Cooper pair subspace forms a two-level system (TLS). Charge states that include one or more quasiparticles, on the other hand, are not single levels. Instead, the quasiparticle momentum states form a continuous band, which, in nanoscale devices, is typically described by the Dynes density of states $[45,46]$. There is therefore a difference between a microwave field driving Rabi oscillation between the discrete states $|G\rangle$ and $|E\rangle$ (described in Sec. IV) and microwaves creating split Cooper pairs to drive the system from $|G\rangle$ or $|E\rangle$ to a $(1,1)$ charge state lying in a continuous band (described in Sec. V). Finally, in Sec. VI, we combine the two transitions to drive Cooper pair splitting at a rate dependent on the relative occupancy of the two states of the Cooper pair charge state subspace. These transitions and the structure of our paper are summarized in Fig. 2. 
(a)

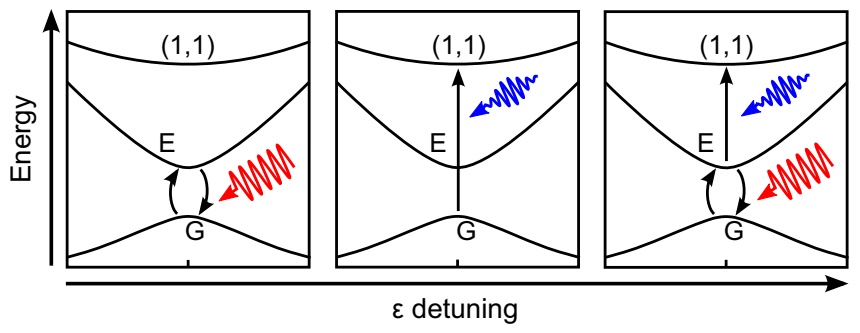

FIG. 2. An outline of our paper. (a) In Sec. IV, we study the resonant excitation of the ground and excited Cooper pair states. (b) In Sec. V, we study Cooper pair breaking driven by higherenergy photons. (c) We combine these two processes in Sec. VI to demonstrate quasiparticle generation dependent on the quantum state of the initial state.

\section{EXPERIMENTAL METHODS}

Our device [Fig. 3(a)] is made by standard three-angle shadow evaporation [47] and consists of two aluminium islands tunnel coupled in series to each other and normal-state $\left(\mathrm{Al}_{0.98} \mathrm{Mn}_{0.02}\right)$ leads. Resistive tunnel junctions are formed between the layers by oxidation for four minutes at an $\mathrm{O}_{2}$ pressure of $0.04 \mathrm{mbar}$ for the interisland tunnel barrier, and 10 minutes at $500 \mathrm{mbar}$ for the tunnel barriers to the leads. The interisland normal-state resistance is $\approx 8.5 \mathrm{k} \Omega$, deduced from $E_{J}$ (measured via microwave spectroscopy; see Sec. IV), $\Delta_{0}$, and the Ambegoakar-Baratoff relationship, $E_{J}=\frac{\Delta_{0} h}{8 e^{2} R_{m}}$. Normal-metal-insulator-superconductor (NIS) junction resistances are chosen to be $\approx 5 \mathrm{M} \Omega$ to suppress quasiparticle poisoning from the leads, and this is confirmed by measurement of the overall normal-state resistance of the device to be $\approx 10 \mathrm{M} \Omega$.

From the high-field stability diagram and measurements under applied bias, we determine the charging energies [38] of the device to be $E_{C 1} \approx 310, E_{C 2} \approx 230$, and $E_{C m} \approx 90 \mu \mathrm{eV}$. The Josephson energy of the middle tunnel junction at $B=0$ is found to be $E_{J} \approx 90 \mu \mathrm{eV}$ by microwave spectroscopy (see Sec. IV) and confirmed by fitting the resulting band structure curvature to the measured capacitance. By measuring Coulomb diamonds as a function of $B$ along $\epsilon$, and spanning several charge stability cells, we determine $\tilde{\Delta}$ as a function of $B$. We find that at $B=0$ and a temperature of $35 \mathrm{mK}$, $\tilde{\Delta}=250 \mu \mathrm{eV}$.

We measure the capacitance of the SDD at $35 \mathrm{mK}$ by radio-frequency reflectometry [Fig. 3(b)]. The device is embedded in a resonant circuit comprising an inductor $\left(L_{r}=470 \mathrm{nH}\right)$ and parasitic capacitance $\left(C_{r}=0.035 \mathrm{pF}\right)$, giving a resonant frequency of $f_{r}=349 \mathrm{MHz}$. A low-power (typically $<-100 \mathrm{dBm}$ ) rf signal close to $f_{r}$ is incident on the resonator, and the amplitude and phase of the reflected power are measured by homodyne detection. We deduce changes in the capacitance of the device from phase changes in the reflected power. Microwave frequency radiation is delivered to the sample via $V_{\mu W}$. Magnetic fields are applied in the plane of the device.

We take care to minimize the nonequilibrium quasiparticle generation in the superconductor by unwanted radiation from (a)

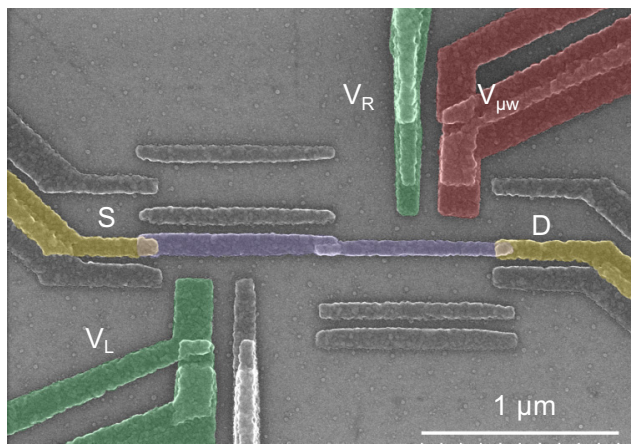

(b)

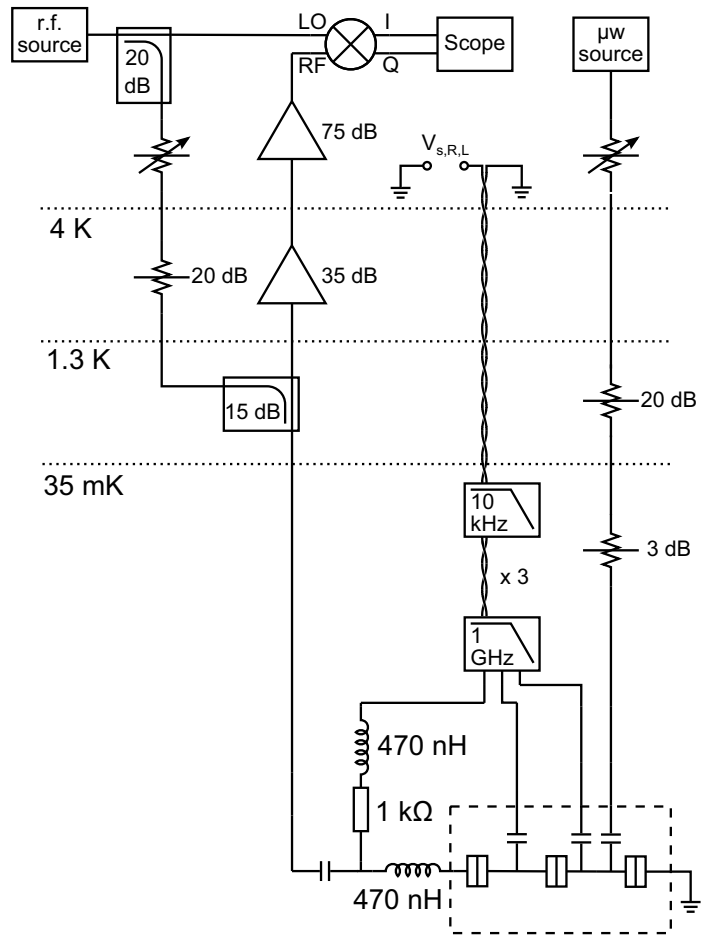

FIG. 3. Device and experimental wiring. (a) False color scanning electron micrograph of SDD. Superconducting islands are in purple; normal-state leads are in yellow. Electrostatic gates are highlighted in green (dc) and red (microwave frequencies). Uncolored features are artifacts of the three-angle evaporation process. (b) Experimental wiring: Reflectometry circuit (left), dc wiring (middle) with low-temperature $R C$ and Eccosorb filtering, and the microwave line (right) with attenuation at $1.4 \mathrm{~K}$ and $35 \mathrm{mK}$.

higher-temperature stages of the dilution refrigerator. The dc gate biases are applied to one-half of a twisted pair, with the other half grounded, forming a distributed $R C$ filter. Lumped element filters at $35 \mathrm{mK}$, with a cutoff frequency of $10 \mathrm{KHz}$, are also used. The twisted pairs are sandwiched for a length of $\approx 120 \mathrm{~mm}$ between two layers of Eccosorb microwave absorbent material, to attenuate higher $(>1 \mathrm{GHz})$ frequencies. The carrier tone for reflectometry is attenuated by $20 \mathrm{~dB}$ at the $4 \mathrm{~K}$ stage of the dilution fridge, and a further $15 \mathrm{~dB}$ at $35 \mathrm{mK}$. Microwave cables are attenuated by $20 \mathrm{~dB}$ at $4 \mathrm{~K}$, and $3 \mathrm{~dB}$ at $35 \mathrm{mK}$. The sample is mounted in a light tight copper box, coated internally with a microwave absorbent coating $[20,48]$. It is then surrounded by more Eccosorb. 


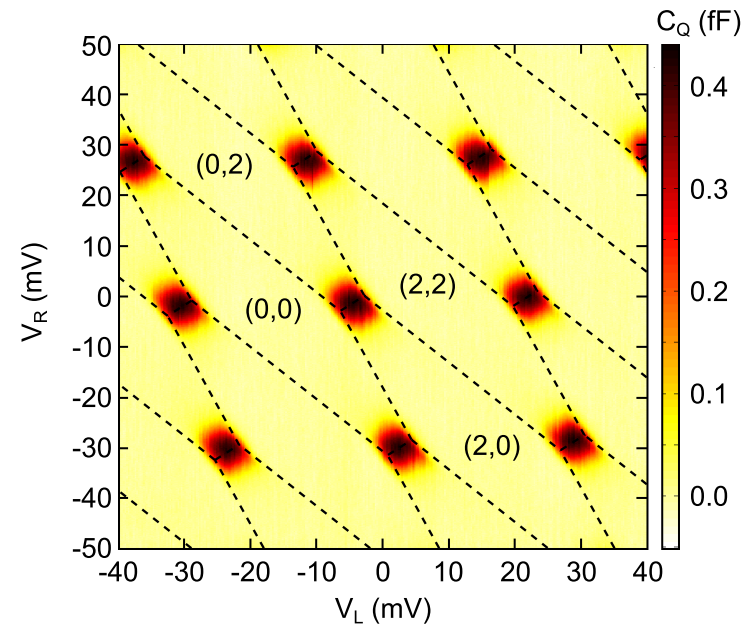

FIG. 4. Measured capacitance of the SDD as a function of $V_{L}$ and $V_{R}$. The quantum capacitance is seen around the interdot charge transitions. Dotted lines highlight the $2 e$ periodic charge stability diagram. Charge states are numbered from an arbitrary even-parity state. Odd-parity states are higher in energy and therefore not observed in the stability diagram.

In Fig. 4, we show the measured capacitance of our device as a function of $V_{L}$ and $V_{R}$, at $B=0$ and $T=35 \mathrm{mK}$. The capacitance change due to the anticrossings between evenparity states can be seen, and the hexagonal charge stability diagram is highlighted. Under a large magnetic field of $B=$ $2 \mathrm{~T}$, the aluminium is in the normal state, and the resulting change in periodicity (not shown) allows us to confirm that the stability diagram in Fig. 4 is $2 e$ periodic.

\section{COHERENT MICROWAVE DRIVING OF THE $G$-E TRANSITION}

We first describe our microwave spectroscopy of the TLS formed by $|E\rangle$ and $|G\rangle$. By observing the quantum capacitance while driving Rabi oscillations, we measure the energy splitting $E_{J}$ between the two states. In Fig. 5, we plot quantum capacitance against $\epsilon$ detuning and frequency of applied microwaves. Two- and three-photon transitions are clearly visible, but the one-photon transition is less clear because the frequency dependence of the transmission of our microwave line limits the power delivery around $23 \mathrm{GHz}$.

We now probe the relaxation time $T_{1}$ of $|E\rangle$ at $\delta=\epsilon=0$. We proceed by saturating the transition with a microwave tone modulated by a symmetric square wave of period $\tau$ [49] and measuring the time-averaged quantum capacitance. The period of the Rabi oscillation of the system is much shorter than the relaxation time and so, at the limit of $\tau_{0} \ll T_{1}$, the time-averaged populations of the states, $p_{G}$ and $p_{E}$, are $p_{G}=$ $p_{E}=1 / 2$ and the measured quantum capacitance averages to zero. For longer $\tau$, there is sufficient time for some decay of $p_{E}$ to occur during the microwave-off period, and a finite quantum capacitance is measured.

In Fig. 6, we plot the normalized change in capacitance under the modulated microwave drive as a function of $\tau$. Two relaxation rates can be seen, one at short-time scales and one at much larger $\tau$. We ascribe the short-time-scale

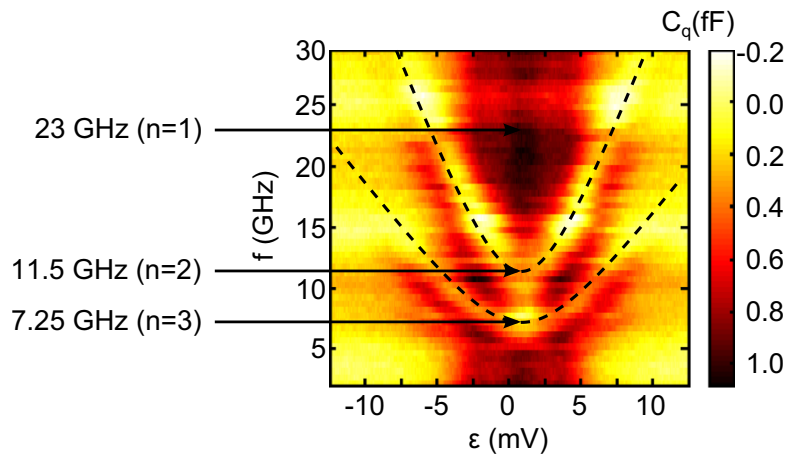

FIG. 5. Quantum capacitance along the $\epsilon$ detuning axis as a function of applied microwave frequency. The nonzero quantum capacitance is seen in the center of this region (black). When the applied photons are resonant with the transition between $|E\rangle$ and $|G\rangle$, Rabi oscillations are driven and the population-weighted quantum capacitance of the two states is zero (yellow regions). Parabolas corresponding to two- and three-photon transitions are seen clearly and are highlighted with dashed lines.

behavior to relaxation of $|E\rangle$ and the long-time-scale dynamics to relaxation from a small population $\left(p_{q p}\right)$ in the $(1,1)$ state. For relaxation involving two possible excited states, the normalized capacitance change is given by

$$
\frac{\delta C}{\delta C_{\max }}=\frac{1}{2}+\frac{p_{E} T_{1}}{\tau}\left(1-e^{-\tau /\left(2 T_{1}\right)}\right)+\frac{p_{q p} T_{q p}}{\tau}\left(1-e^{-\tau /\left(2 T_{q p}\right)}\right),
$$

where $T_{q p}$ is the relaxation time of the quasiparticle state. We fit Eq. (2) to the data in Fig. 6, giving $T_{1}=(6.8 \pm$ $1.0) \times 10^{-9} \mathrm{~s}$. This is much shorter than for charge states in optimized semiconductor double dots $\left(T_{1} \sim 0.01-100 \mu \mathrm{s}\right)$ [50], superconducting charge qubits $\left(T_{1} \sim 1 \mu \mathrm{s}\right)$ [51], and transmons $\left(T_{1} \sim 40 \mu \mathrm{s}\right)$ [9] due to the large dipole moment

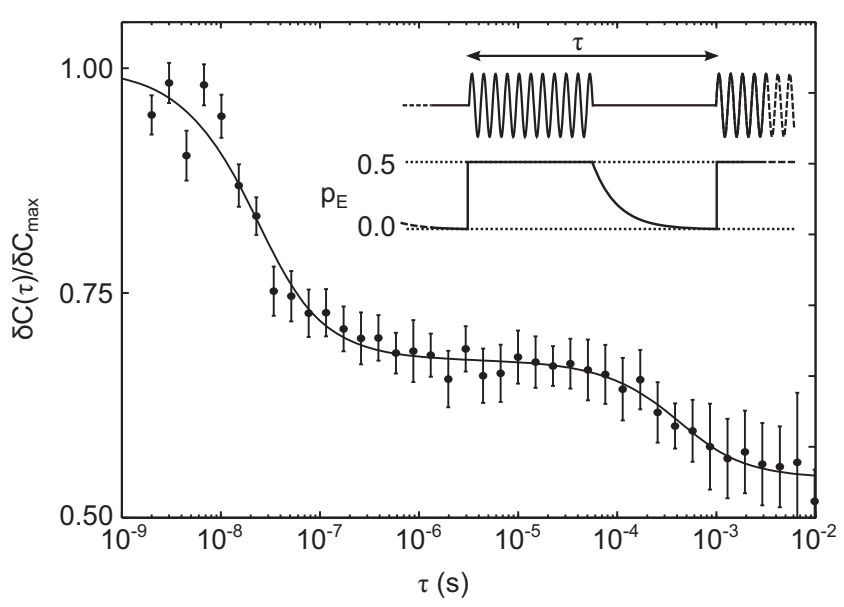

FIG. 6. Normalized change in capacitance $\delta C(\tau) / \delta C_{\max }$ under symmetrically pulsed $11.5 \mathrm{GHz}$ microwave excitation (inset, along with the resulting $p_{E}$ ), as a function of pulse period $\tau$, with a fit to Eq. (2). Two relaxation times are observed: a fast relaxation of $p_{E}$ with $T_{1}=(6.8 \pm 1.0) \times 10^{-9} \mathrm{~s}$ and a slower relaxation of $p_{q p}$ with time constant $T_{q p}=(1.2 \pm 0.4) \times 10^{-4} \mathrm{~s}$. 
associated with charge transitions between the $\sim 1 \mu \mathrm{m}$ islands, and the strong coupling between them.

We find that $T_{q p}=(1.2 \pm 0.4) \times 10^{-4} \mathrm{~s}$, which is comparable to the times measured in SSETs [52], and on these time scales, real-time measurement of splitting and recovery of single Cooper pairs is possible [31]. We study this in the next section.

\section{COOPER PAIR BREAKING BY INCOHERENT PHOTON ABSORPTION}

We now study the interaction of the SDD with radiation of higher frequencies, significantly detuned from the $23 \mathrm{GHz}$ resonance between $|G\rangle$ and $|E\rangle$. Microwaves of frequencies up to $55 \mathrm{GHz}$ are introduced via the gate capacitor and we measure the capacitance of the device in real time. Traces are taken over an interval of $10 \mathrm{~s}$ with a bandwidth of $150 \mathrm{kHz}$. We observe asymmetric random telegraph signals (RTSs), indicative of stochastic switching between two states [Fig. 7(a)]. Elsewhere [31], we have studied this process in the absence of an applied microwave drive and, by studying the $\delta$-detuning dependence, established that Cooper pairs are being broken to the $(1,1)$ state with relaxation occurring via single-quasiparticle states such as $(1,0)$, mediated by Andreev processes and tunneling to the leads. Here, we concentrate on the behavior under varying frequencies $(f)$ and powers $(P)$ of microwave irradiation at zero $\epsilon$ and $\delta$ detuning.

Our RTS is characterized by the rates $\Gamma_{G \rightarrow(1,1)}$, the rate of broken pair creation, and $\Gamma_{\rightarrow G}$, the rate at which the system returns to the ground state. $\Gamma_{G \rightarrow(1,1)}$ increases linearly with $P$, suggesting that a single-photon process is responsible. We parametrize the microwave sensitivity of the SDD by $\frac{d \Gamma_{G \rightarrow(1,1)}}{d P}$ [Fig. 7(b)]. The microwave sensitivity is strongly dependent on the applied magnetic field; at low fields it has a constant value but increases exponentially at higher fields, as shown in Fig. 7(c) where the sensitivity is plotted on a logarithmic scale.

As the magnetic field is increased, it suppresses $\tilde{\Delta}$, reducing the energy difference between the $(1,1)$ and ground states $\left(E_{(1,1)-G}\right)$. This increases the density of states in the region within $h f$ of the energy of state $|G\rangle$ [Fig. 7(c) inset]. The rate of broken pair creation due to electromagnetic radiation is given by [53]

$$
\Gamma \propto \int_{0}^{h f+\frac{E_{c}}{2}} R_{\epsilon} N(\epsilon) N\left(h f+\frac{E_{c}}{2}-\epsilon\right) d \epsilon .
$$

Here, $h f$ is the photon energy, $N(\epsilon)$ is the Dynes density of states [45], $R_{\epsilon}$ is the case-II coherence factor corresponding to a perturbation that is odd under time reversal [53], and the additional term involving $\frac{E_{c}}{2}$ is due to the additional energy available from charge reconfiguration in the device. In Fig. 7(c), we also show a fit of Eq. (3) to the data, with the Dynes broadening parameter $\gamma=0.014$. Here we do not include the data point for $B=200 \mathrm{mT}$ in the fit, as in this regime transitions to quasiparticle states other than $(1,1)$ become significant processes. Our model replicates the above-exponential rate of increase of sensitivity well, and similar behavior is seen for other frequencies.

Having established the effect of a magnetic field on the energy structure of the SDD, we now remove the applied
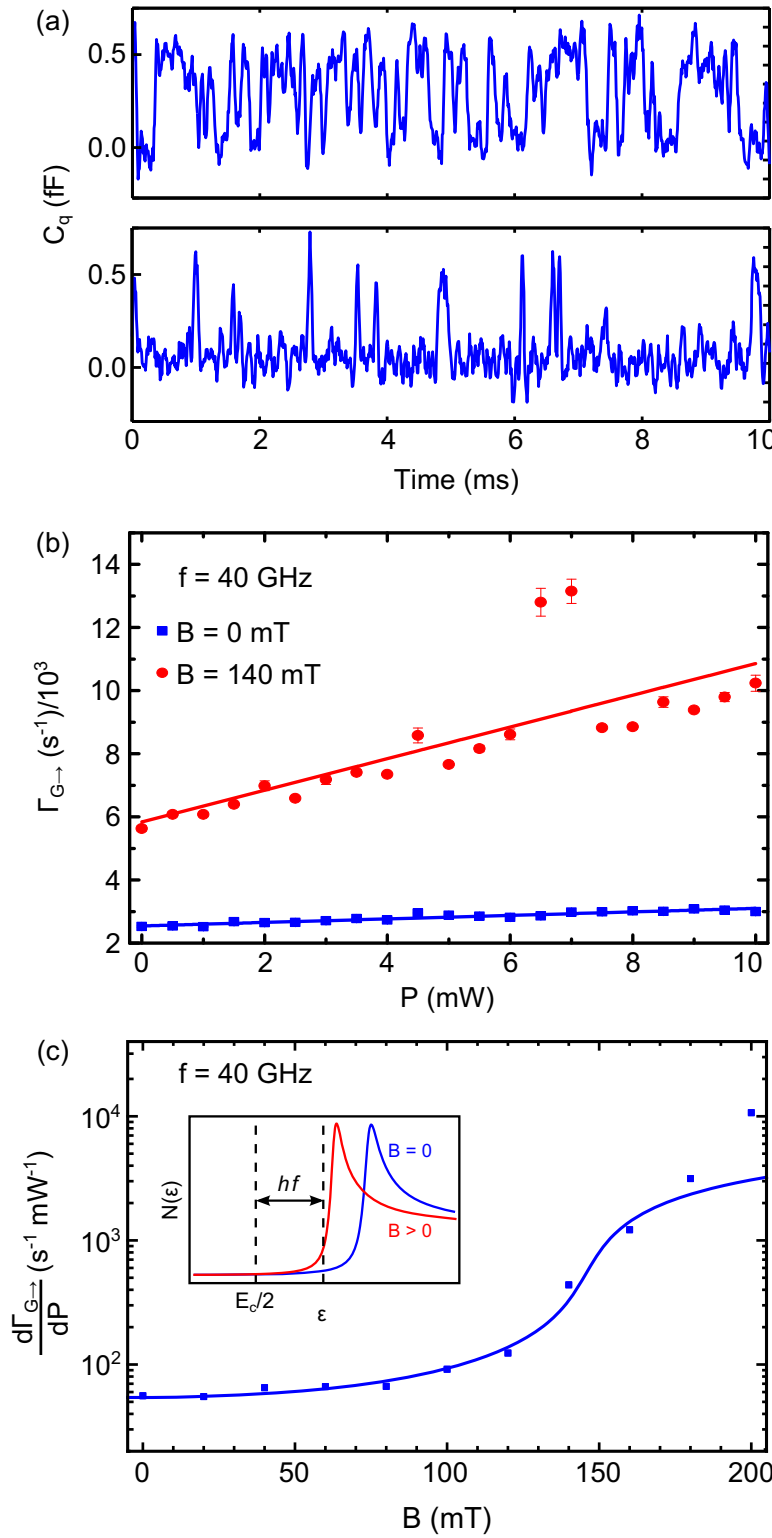

FIG. 7. Breaking of Cooper pairs by microwave radiation. (a) Real-time capacitance measurements for $40 \mathrm{GHz}$ microwaves with an applied in-plane field of $B=140 \mathrm{mT}$. For the top panel, the microwave power at the source was $1 \mathrm{~mW}$, and for the lower panel, it was $20 \mathrm{~mW}$. RTSs with different transition rates are seen. (b) Breaking rate $\Gamma_{G \rightarrow(1,1)}$ as a function of microwave power for $40 \mathrm{GHz}$ radiation, and applied fields of $B=0$ and $B=140 \mathrm{mT}$. The gradient of the linear fit, $\frac{d \Gamma_{G \rightarrow(1,1)}}{d P}$, parameterizes the sensitivity of the device to radiation. (c) $\frac{d \Gamma_{G \rightarrow(1,1)}}{d P}$ as a function of magnetic field $B$ for $40 \mathrm{GHz}$ microwaves, with a fit to Eq. (3). Note the logarithmic scale for the $y$ axis. The inset cartoon shows the increase in DOS in the region accessible to photons of energy $h f$ due to the decrease in $\tilde{\Delta}$.

microwave drive and turn our attention to the rate of pair splitting due to the cryogenic environment of the device. We have demonstrated that the breaking rate due to photons of a particular frequency is dependent on the energy required to create a split pair, $E_{(1,1)-G}$. By tuning $E_{(1,1)-G}$, we can perform spectroscopy on the photons that cause pair splitting. We make the approximation that photons with energy $h f>E_{(1,1)-G}$ are 
(a)

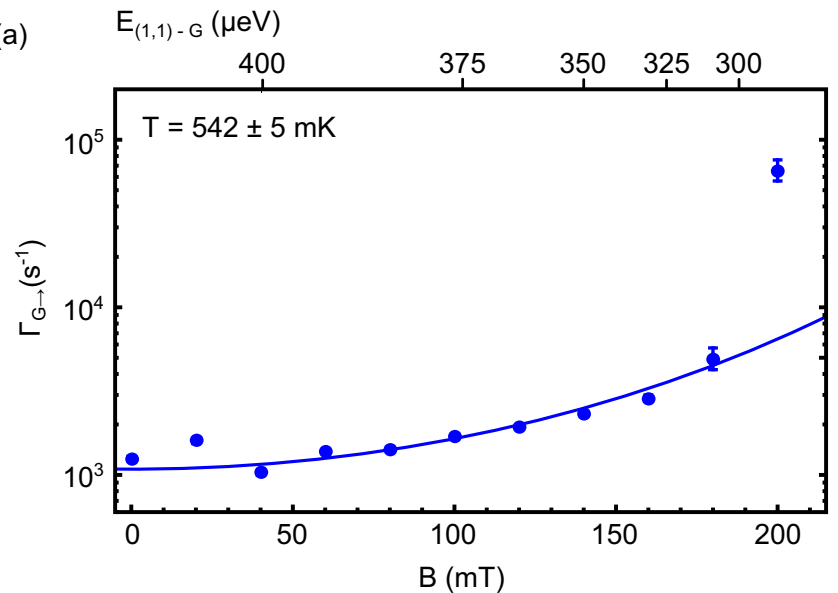

(b)

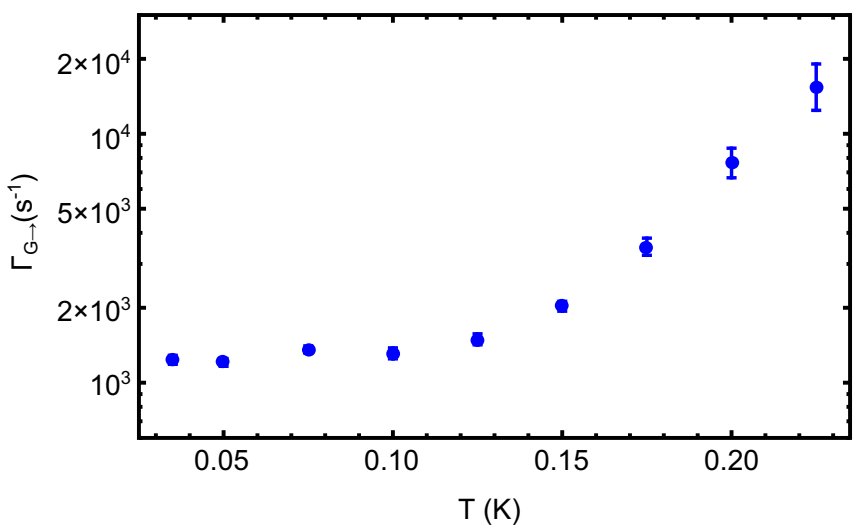

FIG. 8. Spectroscopy on the ambient thermal radiation in the sample space. (a) $\Gamma_{G \rightarrow(1,1)}$ vs magnetic field. On the top $x$ axis, we show the value of $E_{(1,1)-G}$ corresponding to the applied field axis. A fit to an integrated Planck spectra with temperature $T=542 \pm 5 \mathrm{mK}$ is also shown. (b) $\Gamma_{G \rightarrow(1,1)}$ as a function of temperature showing a low-temperature saturation at about $125 \mathrm{mK}$.

able to create a quasiparticle pair, and photons with energy $h f<E_{(1,1)-G}$ are not. Thus,

$$
\Gamma_{G \rightarrow(1,1)}=A \int_{E_{(1,1)-G}}^{\infty} n(E) d E,
$$

where $n(E)$ is the number density of pair splitters with a given energy and $A$ is a constant.

In Fig. 8(a), we plot our measurements of $\Gamma_{G \rightarrow(1,1)}$ as a function of $B$, and also display the values of $E_{(1,1)-G}$ corresponding to the applied field values on a second $x$ axis, as determined from the measured device parameters (see Sec. III). We find a good fit to our data by Eq. (4) using a blackbody spectrum for $n(E)$, with a temperature of $T=542 \pm 5 \mathrm{mK}$. Here, as before, we neglect data at $B=200 \mathrm{mT}$. The most likely candidate for the source of this thermal noise is Johnson noise from the $20 \mathrm{~dB}$ attenuator on the microwave line at $1.3 \mathrm{~K}$, subsequently attenuated by approximately a factor of two by the $3 \mathrm{~dB}$ attenuator at $35 \mathrm{mK}$ [54].

It is also possible to reduce $E_{(1,1)-G}$ by increasing the temperature of the SDD due to the thermodynamic suppression of $\tilde{\Delta}$. This is primarily because of the linear dependence of the second term of Eq. (1) on $T$, rather than the slower increase in $\ln \left(N_{\text {eff }}\right)$. In Fig. 8(b), we plot the microwave-off temperature dependence of $\Gamma_{G \rightarrow(1,1)}$, finding that it is constant until a temperature of approximately $125 \mathrm{mK}$. We interpret this as the base electron temperature of the SDD's environment, elevated from the measured temperature of the mixing chamber due to imperfect shielding and filtering [20,55]. Above this temperature, $\Gamma_{G \rightarrow(1,1)}$ increases rapidly as $\tilde{\Delta}$ decreases.

\section{ASSISTED COOPER PAIR BREAKING}

To recap, the key states in our device are a two-level manifold of even-parity Cooper pair states, $|G\rangle$ and $|E\rangle$, and a continuum of doubly odd-parity quasiparticle states with the maximum in the Dynes density of states (corresponding to the BCS band edge) having higher energies than both even-parity states. In this section, to confirm our understanding of the SDD and increase its sensitivity, we enhance the rate of Cooper pair splitting by preparing the Cooper pair with a significant population in the higher-energy state $|E\rangle$. When the system is in state $|E\rangle$, the energy required to reach the quasiparticle band edge is reduced by $E_{J}$.

To measure the effect on the splitting rate of different populations of $|E\rangle$, we need to determine the population of $|E\rangle$ for a given Rabi tone power and the breaking rate with and without each tone. To achieve this, we apply the following pulse sequence [Fig. 9(a)] and measure the phase response [Fig. 9(b)], averaging the measurement over $3 \times 10^{4}$ repetitions.

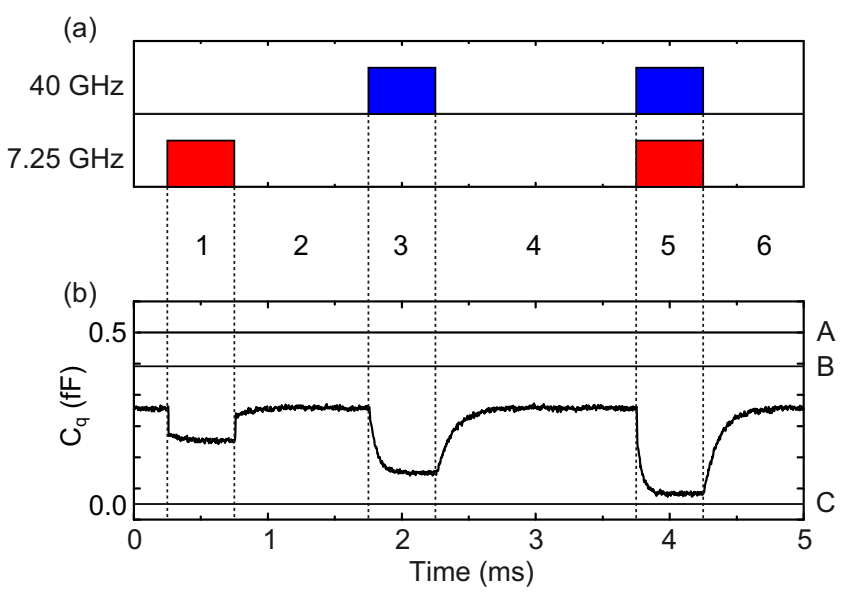

FIG. 9. Scheme for measuring state-dependent Cooper pair splitting. (a) Envelopes of applied microwave pulses as a function of time. $40 \mathrm{GHz}$ pulses intended to split Cooper pairs are shown in the top row and $7.25 \mathrm{GHz}$ pulses to populate the antisymmetric Cooper pair state are shown in the bottom row. (b) Measured capacitance averaged over $3 \times 10^{4}$ repetitions. $7.25 \mathrm{GHz}$ radiation populates the antisymmetric Cooper pair state on time scales that are much faster than our measurement bandwidth. The change of capacitance due to Cooper pair splitting driven by the $40 \mathrm{GHz}$ radiation shows as a slower exponential decay in capacitance. From analysis of RTSs, we associate the capacitance marked A with the ground state $|G\rangle$, B with the weighted mixture of $|E\rangle$ and $|G\rangle$ due to the Rabi tone $(0.08 \mathrm{~mW}$ in this plot), and $\mathrm{C}$ with the $(1,1)$ state. There is a significant $(1,1)$ population in all sections, so the measured capacitance is a weighted average of $\mathrm{A}$ or $\mathrm{B}$, and $\mathrm{C}$. 
(1) A Rabi tone alone, at $7.25 \mathrm{GHz}$, is applied. The antisymmetric state is populated via three-photon transitions, giving a sharp drop in capacitance on the time scale of the Rabi oscillations, and some pair breaking occurs.

(2) The Rabi tone is switched off. The antisymmetric population relaxes rapidly $(\sim \mathrm{ns})$ and the broken pair population relaxes more slowly (See Sec. IV.)

(3) A breaking tone alone is applied, resulting in a moderate rate of Cooper pair breaking.

(4) The breaking tone is switched off and the broken pair population relaxes.

(5) Both Rabi and breaking tones are applied simultaneously. The antisymmetric state is populated on short-time scales, followed by faster pair breaking.

(6) Both tones are switched off. The antisymmetric population relaxes rapidly and the broken pair population relaxes slowly.

We choose $7.25 \mathrm{GHz}$ for the Rabi tone in order to suppress direct pair breaking driven by this tone. Breaking decays approximately exponentially as frequency is reduced from the band edge, whereas the power required for $n$-photon transitions to populate $|E\rangle$ drops off as a power law. Here the breaking remains dominated by the thermal background. $40 \mathrm{GHz}$ is chosen for the frequency of the breaking pulse.

We assume no time dependence of $\frac{p_{G}}{p_{G}+p_{E}}$ and $\frac{p_{E}}{p_{G}+p_{E}}$ within each section of the series, as $p_{E}$ saturates on a much shorter time scale than that of our measurement. The time-dependent split state population $p_{q p}(t)$ in each section is given by

$$
\frac{d p_{q p}(t)}{d t}=\Gamma_{\{G, E\} \rightarrow(1,1)}\left[1-p_{q p}(t)\right]-\Gamma_{\rightarrow\{G, E\}} p_{q p}(t) .
$$

This is readily solved to give

$$
p_{q p}(t)=\frac{\Gamma_{\{G, E\} \rightarrow(1,1)}}{\Gamma_{\rightarrow\{G, E\}}+\Gamma_{\{G, E\} \rightarrow(1,1)}}+C e^{-\left(\Gamma_{\rightarrow\{G, E\}}+\Gamma_{\{G, E\} \rightarrow(1,1) t}\right.} .
$$

$C$ is the constant of integration and we have relabeled the rates $\Gamma$ to emphasize that both $|E\rangle$ and $|G\rangle$ are now populated.

To convert the time trace in Fig. 9(b) to the broken pair population, it is necessary to know the capacitance change associated with the quasiparticle state. To determine both this and $p_{E}$ under microwave driving, we take two $10 \mathrm{~s}$ time traces for each power level: one with the Rabi pulse on and one with it off. Each shows a random telegraph signal, and to accurately determine the capacitances of the two states we use the techniques described in the Appendix.

To measure the efficacy of the state-dependent splitting, we repeat this for different breaking tone and Rabi tone power levels. In Fig. 10(a), we show $\Gamma_{\{G, E\} \rightarrow(1,1)}$ as a function of breaking tone power for both Rabi tone on and off. The breaking rate is linear with probe power, as in Sec. V above, for both Rabi tone on and off. However, by switching on the Rabi tone, we have increased the sensitivity (parameterized by $\left.\frac{d \Gamma_{\{G, E \rightarrow(1,1)}}{d P_{\text {probe }}}\right)$ by $\sim 80 \%$. In Fig. 10(b), we confirm that this increase in sensitivity is due to the enhanced population of $|E\rangle$ by showing $\Gamma_{\{G, E\} \rightarrow(1,1)}$ as a function of preparation power. As the Rabi tone power increases, the population of $|E\rangle$ increases. This population can be estimated from the quantum capacitance signal associated with the Cooper pair manifold
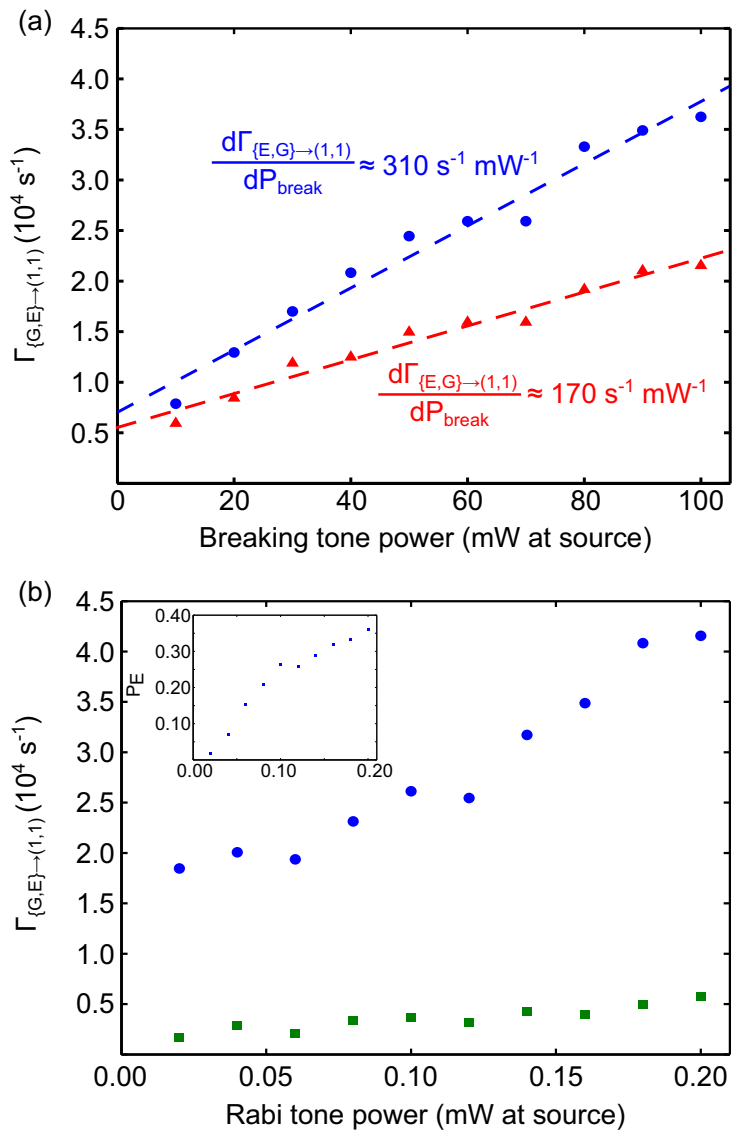

FIG. 10. Quasiparticle generation rates under two frequencies of irradiation. (a) Generation rate under $40 \mathrm{GHz}$ driving only (red triangles) and simultaneous driving with $0.08 \mathrm{~mW}, 7.25 \mathrm{GHz}$, and $40 \mathrm{GHz}$ tones (blue circles) as a function of microwave power of the $40 \mathrm{GHz}$ tone. (b) Generation rate under $7.25 \mathrm{GHz}$ driving only (green squares) and simultaneous driving with $0.08 \mathrm{~mW}, 7.25 \mathrm{GHz}$, and $40 \mathrm{GHz}$ tones (blue circles) as a function of microwave power of the $7.25 \mathrm{GHz}$ tone.

[inset to Fig. 10(b)]. By increasing the population of the excited state, we increase $\Gamma_{\{G, E\} \rightarrow(1,1)}$ and, with it, the sensitivity of our device to the breaking tone.

\section{CONCLUSION}

The SDD is a system with a rich range of dynamic behavior, and here we have explored the interactions of the charge states with microwave frequency radiation, allowing us to characterize the band structure and charge coherence of the TLS.

Our principal result is the splitting of Cooper pairs with higher-frequency radiation and, in contrast to previous devices [56-59], we retain the split pair on our device. Such a pair would be entangled and quasiparticles in aluminium have long spin life times [60] and could therefore be a potential resource for quantum technologies. Owing to the large dispersive shift between the ground and $(1,1)$ states, an SDD can be used as a tunable passive click detector for microwave frequency radiation in the 20 to $55 \mathrm{GHz}$ range, and we demonstrate that it can be used to probe its environment and provide an 
understanding of the spectrum of pair breakers. This also is an important frequency range for radioastronomy studies of the reionization era [61], with the Square Kilometer Array, the Atacama Large Millimetre Array, and Planck operating detectors in this bandwidth. We also demonstrate that the sensitivity of the SDD can be tuned by exploiting the presence of the Cooper pair manifold. By selectively populating $|E\rangle$, we increase the rate of pair breaking under incident radiation.

Finally, we note that our device is straightforward to integrate with antennas, for an array of detectors, or with existing superconducting quantum devices including superconducting resonators and embedded qubits, offering a possible path towards entanglement by measurement in a circuit QED platform.

\section{ACKNOWLEDGMENTS}

We acknowledge the support from Hitachi Cambridge Laboratory and EPSRC Grants No. EP/K027018/1 and No. EP/K025562. A.J.F. was financially supported by a Hitachi Research fellowship.

\section{APPENDIX: ANALYSIS OF RANDOM TELEGRAPH SIGNALS}

Processes involving Markovian switching between two states often result in the experimental measurement of an asymmetric random telegraph signal (RTS). An RTS is characterized by a signal switching between two levels $a_{0}$ and $a_{1}$, with a switching rate from $a_{0}$ to $a_{1}\left(a_{1}\right.$ to $\left.a_{0}\right)$ of $\Gamma_{0(1)}$. In Secs. V and VI, time-domain measurements of capacitance yield random telegraph signals due to stochastic switching between charge states. Analysis of such a signal generally focuses on extracting the rates $\Gamma_{0,1}$ and, for a signal far from the limits of measurement with a noise amplitude $a_{n} \ll a_{1}-a_{0}$ and a measurement bandwidth $1 / \tau \gg \Gamma_{0}+$ $\Gamma_{1}$, this is straightforward; the individual time periods for which the signal remains above or below some threshold are histogrammed and an exponential decay is fitted to the resulting points.

If these conditions do not obtain, more sophisticated techniques must be used. In the case where the noise amplitude becomes similar to the signal, the signal may cross the threshold due to noise and time periods may be incorrectly broken by an excursion to the wrong level. In this case, the previous method can be recovered to a certain extent by use of a Schmidt trigger, in which separate thresholds for transitions from $a_{0}$ and $a_{1}$ are used. Alternatively, joint time-frequency wavelet edge detection methods can be used [62] or the signal can be analyzed purely in the frequency domain. The power spectrum of an RTS is given by [63]

$$
S_{\mathrm{RTS}}(\omega)=\frac{4\left(a_{1}-a_{0}\right)^{2}}{\left(\Gamma_{0}^{-1}+\Gamma_{1}^{-1}\right)\left[\left(\Gamma_{0}+\Gamma_{1}\right)^{2}+\omega^{2}\right]},
$$

but the spectrum is symmetric with respect to $\Gamma_{0}$ and $\Gamma_{1}$, and the two cannot be separated.

If the bandwidth of the measurement is not much greater than $\Gamma_{0}+\Gamma_{1}$, then a genuine short-lived period in a particular
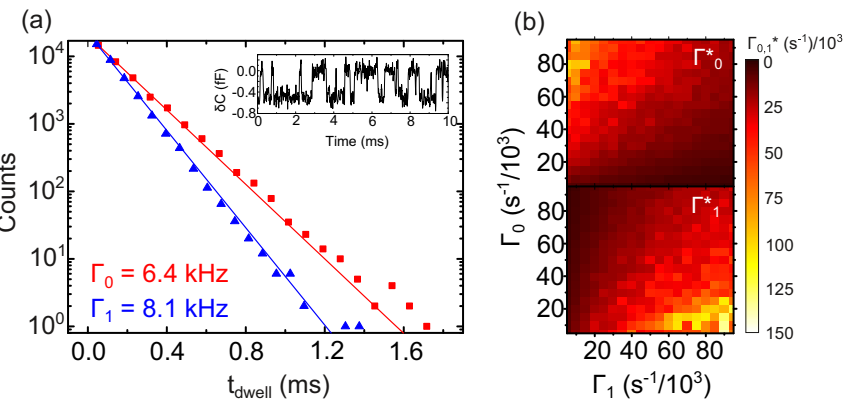

FIG. 11. Conventional analysis of RTSs. (a) Histogram of dwell times resulting from dividing the time-domain measurement (inset) into "up" and "down" periods. Exponential decays are fitted to the two sets of data, giving measured times $\Gamma_{0,1}^{*}$. (b) Calibration data for converting $\Gamma_{0,1}^{*}$ to $\Gamma_{0}$ and $\Gamma_{1}$.

state may be missed [64]. The effect here is twofold: it removes a short-time period that should have been counted and it joins two longer-time periods together into one.

These difficulties result in measured rates $\Gamma_{0}^{*}$ and $\Gamma_{1}^{*}$, which are much less than the underlying ones. Here we overcome these problems by calibrating our measurement algorithm against prepared RTSs of known rates, with the noise amplitude fixed to be the same as in our measurements. Signals are digitally synthesized by an AWG at $1.2 \times 10^{9}$ samples per second, well above our measurement bandwidth which is fixed
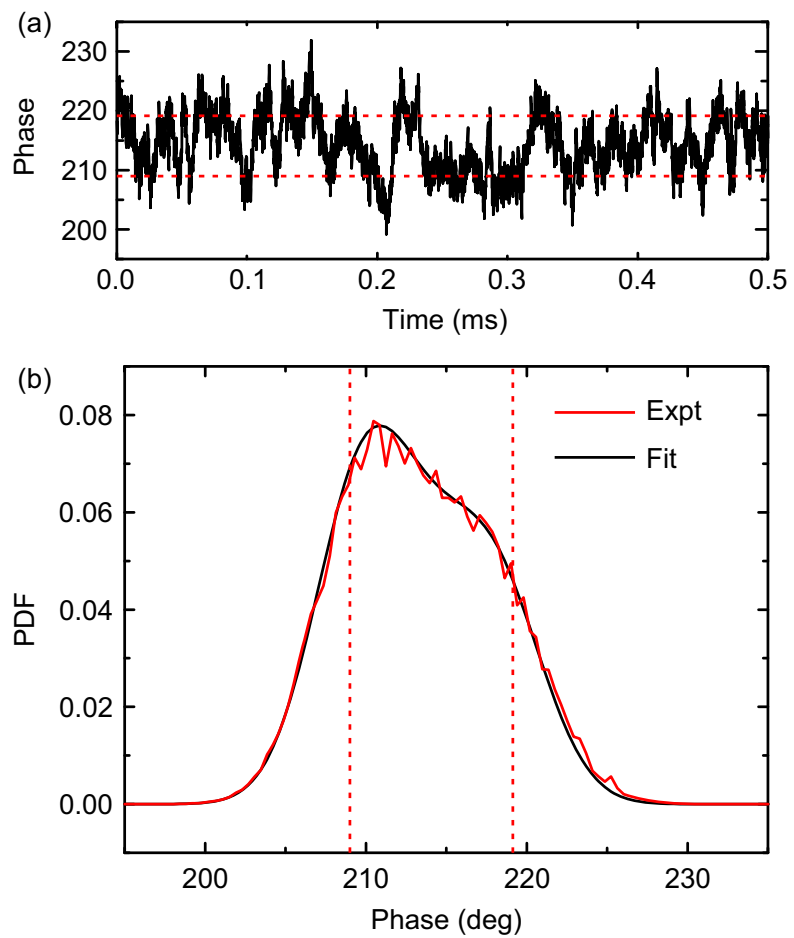

FIG. 12. Analysis of RTS with low signal-to-noise ratio (SNR). (a) A short section of a typical low SNR RTS. (b) The PDF for a $10 \mathrm{~s}$ signal (red) with a fit to Eq. (A2) (black). The levels $a_{0}$ and $a_{1}$, marked with dashed red lines in both (a) and (b), are not coincident with the peaks of the PDF. 
by low-pass filters to be $1 / \tau=150 \mathrm{kHz}$. Results are shown in Fig. 11, showing the interdependence of the measured rates on both $\Gamma_{0}$ and $\Gamma_{1}$.

We also find it necessary (in Sec. VI) to accurately determine $a_{0}$ and $a_{1}$. To do this, we use the probability density function (PDF) of a filtered noise-free RTS [65],

$$
f(y)= \begin{cases}q_{0} f_{0}(y)+q_{1} f_{1}(y) & a_{0} \leqslant y \leqslant a_{1} \\ 0 & \text { otherwise, }\end{cases}
$$

with

$$
f_{0}(y)=\frac{a^{-1} \eta_{0}^{u_{0}-1} \eta_{1}^{u_{1}}}{B\left(u_{0}, u_{1}+1\right)}, \quad f_{1}(y)=\frac{a^{-1} \eta_{0}^{u_{0}} \eta_{1}^{u_{1}-1}}{B\left(u_{0}+1, u_{1}\right)}
$$

Here, $B(g, h)$ is the Beta function, $a_{0}\left(a_{1}\right)$ is the lower (upper) level of the RTS, $a=a_{1}-a_{0}$, and $\eta_{j}=\left|y-a_{j}\right| / a$. $u_{j}=\Gamma_{j} \tau$, with $\tau$ being the measurement bandwidth. To include the effect of experimental noise, we convolve this expression with a Gaussian of width $a_{n}$. We then fit the resulting expression to the PDF of the measured RTS.

In Fig. 12(a), we show a short section of a typical RTS with a low signal-to-noise ratio of $\sim 3$ and and low bandwidthto-rate ratios of $\tau / \Gamma<3$. Figure 12(b) shows the normalized histogram of the entire signal $\left(10^{6}\right.$ data points $)$ in red, with a fit to Eq. (A2) in black. The extracted levels $a_{0}$ and $a_{1}$ of the RTS are highlighted as dashed lines, illustrating the difference between these and the peaks of the PDF.
[1] J. Q. You and F. Nori, Nature (London) 474, 589 (2011).

[2] B. R. Johnson, M. D. Reed, A. A. Houck, D. I. Schuster, L. S. Bishop, E. Ginossar, J. M. Gambetta, L. DiCarlo, L. Frunzio, S. M. Girvin, and R. J. Schoelkopf, Nat. Phys. 6, 663 (2010).

[3] J. M. Martinis, S. Nam, J. Aumentado, and C. Urbina, Phys. Rev. Lett. 89, 117901 (2002).

[4] J. Koch, T. M. Yu, J. Gambetta, A. A. Houck, D. I. Schuster, J. Majer, A. Blais, M. H. Devoret, S. M. Girvin, and R. J. Schoelkopf, Phys. Rev. A 76, 042319 (2007).

[5] J. Clarke and F. K. Wilhelm, Nature (London) 453, 1031 (2008).

[6] J. A. Schreier, A. A. Houck, J. Koch, D. I. Schuster, B. R. Johnson, J. M. Chow, J. M. Gambetta, J. Majer, L. Frunzio, M. H. Devoret, S. M. Girvin, and R. J. Schoelkopf, Phys. Rev. B 77, 180502 (2008).

[7] T. W. Larsen, K. D. Petersson, F. Kuemmeth, T. S. Jespersen, P. Krogstrup, J. Nygård, and C. M. Marcus, Phys. Rev. Lett. 115, 127001 (2015).

[8] Y. Nakamura, Y. A. Pashkin, and J. S. Tsai, Nature (London) 398, 786 (1999).

[9] R. Barends, J. Kelly, A. Megrant, D. Sank, E. Jeffrey, Y. Chen, Y. Yin, B. Chiaro, J. Mutus, C. Neill, P. O’Malley, P. Roushan, J. Wenner, T. C. White, A. N. Cleland, and J. M. Martinis, Phys. Rev. Lett. 111, 080502 (2013).

[10] K. Inomata, Z. Lin, K. Koshino, W. D. Oliver, J.-S. Tsai, T. Yamamoto, and Y. Nakamura, Nat. Commun. 7, 12303 (2016).

[11] A. Wallraff, D. I. Schuster, A. Blais, L. Frunzio, J. Majer, S. Kumar, S. M. Girvin, and R. J. Schoelkopf, Nature (London) 431, 162 (2004).

[12] K. D. Irwin and G. C. Hilton, in Topics in Applied Physics, edited by C. Enss (Springer-Verlag, Berlin, 2005), Vol. 99, pp. 63-149.

[13] P. K. Day, H. G. Leduc, B. A. Mazin, A. Vayonakis, and J. Zmuidzinas, Nature (London) 425, 817 (2003).

[14] C. M. Natarajan, M. G. Tanner, and R. H. Hadfield, Supercond. Sci. Technol. 25, 063001 (2012).

[15] S. Hansjörg and C. Benedetta, Meas. Sci. Technol. 23, 124010 (2012).

[16] J. Zmuidzinas and P. L. Richards, Proc. IEEE 92, 1597 (2004).

[17] S. D. Barrett and P. Kok, Phys. Rev. A 71, 060310 (2005).

[18] H. Bernien, B. Hensen, W. Pfaff, G. Koolstra, M. S. Blok, L. Robledo, T. H. Taminiau, M. Markham, D. J. Twitchen, L. Childress, and R. Hanson, Nature (London) 497, 86 (2013).
[19] E. Knill, R. Laflamme, and G. J. Milburn, Nature (London) 409, 46 (2001).

[20] R. Barends, J. Wenner, M. Lenander, Y. Chen, R. C. Bialczak, J. Kelly, and E. Lucero, Appl. Phys. Lett. 99, 113507 (2011).

[21] A. Kemppinen, S. V. Lotkhov, O. P. Saira, A. B. Zorin, J. P. Pekola, and A. J. Manninen, Appl. Phys. Lett. 99, 142106 (2011).

[22] J. Govenius, R. E. Lake, K. Y. Tan, and M. Möttönen, Phys. Rev. Lett. 117, 030802 (2016).

[23] S. V. Lotkhov and A. B. Zorin, Appl. Phys. Lett. 100, 242601 (2012).

[24] C. J. Chunnilall, I. P. Degiovanni, S. Kück, I. Müller, A. G. Sinclair, Opt. Eng. 53, 081910 (2014).

[25] B. Jalali-Jafari, S. Lotkhov, and A. Zorin, Appl. Sci. 6, 35 (2016).

[26] R. M. Lutchyn, L. I. Glazman, and A. I. Larkin, Phys. Rev. B 74, 064515 (2006).

[27] G. Catelani, J. Koch, L. Frunzio, R. J. Schoelkopf, M. H. Devoret, and L. I. Glazman, Phys. Rev. Lett. 106, 077002 (2011).

[28] J. F. Schneiderman, P. Delsing, G. Johansson, M. D. Shaw, H. M. Bozler, and P. M. Echternach, AIP Conf. Proc. 850, 931 (2006).

[29] G. Catelani, S. E. Nigg, S. M. Girvin, R. J. Schoelkopf, and L. I. Glazman, Phys. Rev. B 86, 184514 (2012).

[30] D. Rainis and D. Loss, Phys. Rev. B 85, 174533 (2012).

[31] N. J. Lambert, M. Edwards, A. A. Esmail, F. A. Pollock, S. D. Barrett, B. W. Lovett, and A. J. Ferguson, Phys. Rev. B 90, 140503 (2014).

[32] N. J. Lambert, A. A. Esmail, M. Edwards, F. A. Pollock, B. W. Lovett, and A. J. Ferguson, Appl. Phys. Lett. 109, 112603 (2016).

[33] T. Hayashi, T. Fujisawa, H. D. Cheong, Y. H. Jeong, and Y. Hirayama, Phys. Rev. Lett. 91, 226804 (2003).

[34] J. R. Petta, A. C. Johnson, J. M. Taylor, E. A. Laird, A. Yacoby, M. D. Lukin, C. M. Marcus, M. P. Hanson, and A. C. Gossard, Science 309, 2180 (2005).

[35] C. Barthel, D. J. Reilly, C. M. Marcus, M. P. Hanson, and A. C. Gossard, Phys. Rev. Lett. 103, 160503 (2009).

[36] B. M. Maune, M. G. Borselli, B. Huang, T. D. Ladd, P. W. Deelman, K. S. Holabird, A. A. Kiselev, I. Alvarado-Rodriguez, 
R. S. Ross, A. E. Schmitz, M. Sokolich, C. A. Watson, M. F. Gyure, and A. T. Hunter, Nature (London) 481, 344 (2012).

[37] S. Gustavsson, M. Studer, R. Leturcq, T. Ihn, K. Ensslin, D. C. Driscoll, and A. C. Gossard, Phys. Rev. Lett. 99, 206804 (2007).

[38] W. G. van der Wiel, S. De Franceschi, J. M. Elzerman, T. Fujisawa, S. Tarucha, and L. P. Kouwenhoven, Rev. Mod. Phys. 75, 1 (2002).

[39] R. Hanson, Rev. Mod. Phys. 79, 1217 (2007).

[40] M. T. Tuominen, J. M. Hergenrother, T. S. Tighe, and M. Tinkham, Phys. Rev. Lett. 69, 1997 (1992).

[41] M. A. Sillanpää, T. Lehtinen, A. Paila, Y. Makhlin, L. Roschier, and P. J. Hakonen, Phys. Rev. Lett. 95, 206806 (2005).

[42] T. Duty, G. Johansson, K. Bladh, D. Gunnarsson, C. Wilson, and P. Delsing, Phys. Rev. Lett. 95, 206807 (2005).

[43] T. Ota, T. Hayashi, K. Muraki, and T. Fujisawa, Appl. Phys. Lett. 96, 032104 (2010).

[44] K. D. Petersson, C. G. Smith, D. Anderson, P. Atkinson, G. A. C. Jones, and D. A. Ritchie, Nano Lett. 10, 2789 (2010).

[45] R. C. Dynes, V. Narayanamurti, and J. P. Garno, Phys. Rev. Lett. 41, 1509 (1978).

[46] J. P. Pekola, V. F. Maisi, S. Kafanov, N. Chekurov, A. Kemppinen, Y. A. Pashkin, O.-P. Saira, M. Möttönen, and J. S. Tsai, Phys. Rev. Lett. 105, 026803 (2010).

[47] G. J. Dolan, Appl. Phys. Lett. 31, 337 (1977).

[48] T. O. Klaassen, J. H. Blok, J. N. Hovenier, G. Jakob, D. Rosenthal, and K. J. Wildeman, in Proceedings of the IEEE 10th International Conference on Terahertz Electronics, Cambridge, UK (IEEE, New York, 2002), pp. 32-35.

[49] J. R. Petta, A. C. Johnson, C. M. Marcus, M. P. Hanson, and A. C. Gossard, Phys. Rev. Lett. 93, 186802 (2004).

[50] K. Wang, C. Payette, Y. Dovzhenko, P. W. Deelman, and J. R. Petta, Phys. Rev. Lett. 111, 046801 (2013).
[51] K. W. Lehnert, K. Bladh, L. F. Spietz, D. Gunnarson, D. I. Schuster, P. Delsing, and R. J. Schoelkopf, Phys. Rev. Lett. 90, 027002 (2003).

[52] A. J. Ferguson, N. A. Court, F. E. Hudson, and R. G. Clark, Phys. Rev. Lett. 97, 106603 (2006).

[53] M. Tinkham, Introduction to Superconductivity: Second Edition (Dover, New York, 2004).

[54] P. J. de Visser, J. J. A. Baselmans, S. J. C. Yates, P. Diener, A. Endo, and T. M. Klapwijk, Appl. Phys. Lett. 100, 162601 (2012).

[55] A. D. Córcoles, J. M. Chow, J. M. Gambetta, C. Rigetti, J. R. Rozen, G. A. Keefe, M. B. Rothwell, M. B. Ketchen, and M. Steffen, Appl. Phys. Lett. 99, 181906 (2011).

[56] L. Hofstetter, S. Csonka, J. Nygård, and C. Schönenberger, Nature (London) 461, 960 (2009).

[57] L. G. Herrmann, F. Portier, P. Roche, A. L. Yeyati, T. Kontos, and C. Strunk, Phys. Rev. Lett. 104, 026801 (2010).

[58] L. Hofstetter, S. Csonka, A. Baumgartner, G. Fülöp, S. d'Hollosy, J. Nygård, and C. Schönenberger, Phys. Rev. Lett. 107, 136801 (2011).

[59] A. Das, Y. Ronen, M. Heiblum, D. Mahalu, A. V. Kretinin, and H. Shtrikman, Nat. Commun. 3, 1165 (2012).

[60] H. Yang, S.-H. Yang, S. Takahashi, S. Maekawa, and S. S. P. Parkin, Nat. Mater. 9, 586 (2010).

[61] P. A. R. Ade et al. (Planck Collaboration), Astron. Astrophys. 571, A16 (2014).

[62] J. R. Prance, B. J. V. Bael, C. B. Simmons, D. E. Savage, M. G. Lagally, M. Friesen, S. N. Coppersmith, and M. A. Eriksson, Nanotechnology 26, 215201 (2015).

[63] M. Kirton and M. Uren, Adv. Phys. 38, 367 (1989).

[64] O. Naaman and J. Aumentado, Phys. Rev. Lett. 96, 100201 (2006).

[65] R. Fitzhugh, Math. Biosci. 64, 75 (1983). 